\title{
Schwierige Erneuerung in Frankreich
}

Mit Emmanuel Macron haben die Franzosen den Kandidaten der Mitte zum Präsidenten gewählt, der die Erneuerung Frankreichs auf seine Fahnen geschrieben hat. Auf inm ruhen große Hoffnungen. Kann er Frankreich aus der Krise und zu alter Stärke führen? Gibt es eine Chance für neuen Schwung in Europa?

Auf Macron wartet eine wahre Herkulesaufgabe. Denn der Wahlkampf, einer der brutalsten der vergangenen Jahrzehnte, hat tiefe Wunden geschlagen, die seine Arbeit erschweren werden. Mehr als $45 \%$ der Wähler haben im ersten Wahlgang links- und rechtsextremistischen Kandidaten ihre Stimme gegeben, die bei allen Differenzen eines gemeinsam haben: die Ablehnung jeglicher pragmatischer, verantwortlicher Regierungspolitik, eine fundamentale Abneigung gegen die Marktwirtschaft, die offene Wirtschaft und Europa. Im zweiten Wahlgang erhielt Marine Le Pen für ihren verquasten national-sozialistischen Kurs immerhin 34\% der Stimmen. Ein Drittel der Wahlberechtigten ist entweder den Urnen ganz ferngeblieben oder hat ungültige Stimmen abgegeben. Viele andere haben Macron nur mit großen Bauchschmerzen gewählt, um die Rechtsextreme Marine Le Pen zu verhindern. Ein Vertrauensvorschuss für den neuen Präsidenten sieht anders aus! All dies ist Ausdruck einer weit verbreiteten Verbitterung bei den Bürgern und einer gespaltenen Gesellschaft. Dazu kommt ein Meinungsklima, das einem simplen klassenkämpferischen Gegensatz zwischen dem "Volk“ und den Eliten bzw. der politischen Klasse konstruiert; ferner ein oft aggressiver Antiliberalismus, der auch vorsichtige marktwirtschaftliche Reformvorhaben als „ultraliberal“ brandmarkt - absurd in einem Land, das die höchste Staatsquote in Europa hat, über einen stark ausgebauten Sozialstaat verfügt und in dem der Staat weiterhin mannigfaltige Interventionsmöglichkeiten besitzt.

Die etablierten bisherigen Regierungsparteien (Sozialisten und Konservative) tragen ein großes Maß an Mitverantwortung an dieser Misere. Keine von ihnen hat in den vergangenen Jahrzehnten den Mut aufgebracht, die Strukturprobleme anzupacken und die Wirtschaft zukunftsfest zu gestalten. Mehr noch: Sie haben sich als abgehobene Kaste verhalten, die sich mit ihren Machtspielen und teilweise ihrer Selbstbedienungsmentalität immer stärker von den Bürgern entfernt und damit die Politikverdrossenheit bis aufs Äußerste gesteigert hat. So ist es kein Wunder, dass sie bei den Wahlen brutal abgestraft worden sind.

Der neue Präsident setzt auf eine Koalition der gemäßigten Kräfte der linken und rechten Mitte, um die notwendigen Reformen in Politik, Wirtschaft und Gesellschaft voranzubringen. Aber kann das gelingen angesichts des verhärteten, polarisierten politischen Klimas? Die Parlamentswahlen werden einen ersten Aufschluss darüber bringen. Die noch sehr junge Bewegung Macrons tritt mit zahlreichen neuen Kandidaten an, deren lokale Verankerung in den Wahlkreisen noch schwach ist. Sie wird es schwer haben, eine Mehrheit zu erringen, und wird wohl Reformkoalitionen mit gemäßigten Kräften der Linken und der Konservativen suchen müssen. Das scheint nicht ausgeschlossen, weil in beiden Lagern gemäßigte Politiker eine derartige Zusammenarbeit befürworten. Auch in der Bevölkerung gibt es laut Meinungsumfragen durchaus Sympathien für eine „Koalition der Willigen“. Daraus könnte sich eine neue Form der Politikgestaltung entwickeln, die weit entfernt ist von dem bisherigen sterilen Gegeneinander zwischen Regierung und Opposition. Dennoch: Ein solches Miteinander der gemäßigten Kräfte kann sich erst allmählich herausbilden in einem Land, das bislang einer klaren Links-Rechts-Polarisierung folgte.

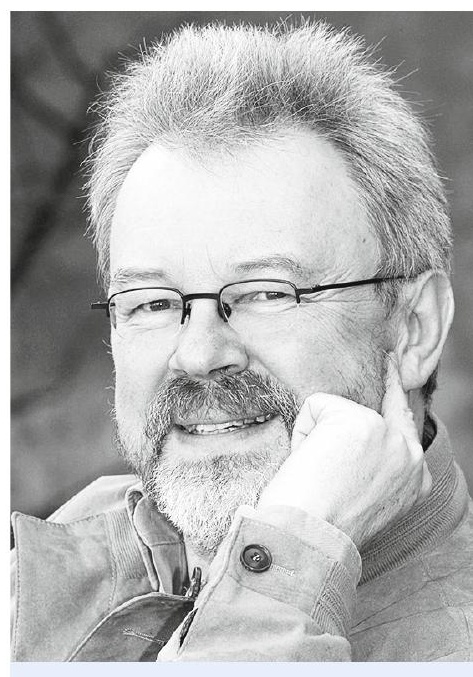

Henrik Uterwedde war stellvertretender Direktor des Deutsch-Französischen Instituts in Ludwigsburg. 
Die Überwindung des Bürgerfrustes und der Radikalisierung des politischen Lebens wird auch von den wirtschaftspolitischen Erfolgen des neuen Präsidenten abhängen. Sein Konzept ist weitgehend schlüssig, weil es an den zentralen bekannten Strukturproblemen Frankreichs ansetzt. Er will erstens das Steuer- und Abgabensystem vereinfachen, die unter Hollande eingeführten Entlastungen der Firmen verstetigen und die Unternehmensteuern von 33,1\% auf 25\% senken. Er tritt für unternehmensfreundlichere Rahmenbedingungen ein und will das Arbeitsrecht vereinfachen, das bislang vor allem beim Mittelstand als Einstellungsbremse fungiert. Zweitens verspricht er Maßnahmen zur Verbesserung der Einkommen breiter Schichten: etwa die Befreiung der Haushalte mit kleinen und mittleren Einkommen von der Wohnraumsteuer (die in vielen Fällen eine hohe Belastung für die Haushalte ist), die Befreiung der Arbeitnehmer von den Beiträgen zur Arbeitslosen- und Krankenversicherung (kompensiert durch eine maßvolle Erhöhung der Sozialversicherungsteuer, die auch Kapitaleinkommen einbezieht) oder verbesserte Leistungen der Krankenversicherung bei Prothesen und Brillen. Drittens setzt der Präsident einen Schwerpunkt auf Bildung und Ausbildung: die Verbesserung der beruflichen Qualifizierung von jungen Arbeitslosen und Langzeitarbeitslosen und vor allem eine grundlegende Reform der beruflichen Bildung mit Aufwertung der dualen Ausbildungsgänge. Für die sozialen Brennpunktviertel, in denen sich soziale Probleme besonders stark zusammenballen, sieht er eine Halbierung der Klassengrößen in der Grundschule vor, um den Kindern dieser Viertel bessere Perspektiven für Schulerfolg und Ausbildung zu geben. Viertens will Macron strukturelle Einsparungen in den öffentlichen Haushalten realisieren, was entsprechende Effizienzsteigerungen und auch Stelleneinsparungen im öffentlichen Dienst voraussetzt. Gleichzeitig soll aber auch eine umfangreiche Investitionsoffensive gestartet werden, um Zukunftsaufgaben wie die berufliche Bildung, die Energiewende oder die Digitalisierung voranzubringen. Fünftens war Macron der einzige Kandidat, der ein eindeutiges Plädoyer für den Ausbau und die Stärkung der europäischen Integration abgegeben hat. Die Vorgaben des Stabilitäts- und Wachstumspakt will er einhalten. Sein Ziel ist es, zunächst durch die Erneuerung der Wirtschaft die verlorengegangene Glaubwürdigkeit Frankreichs zurückzugewinnen, um dann den Partnern, allen voran Deutschland, seine Vorschläge für neue Initiativen in der EU und der Eurozone vorzutragen.

Trotz seiner Ausgewogenheit ist Macrons Programm unpopulär. Der Präsident wird politischen, aber auch heftigen sozialen Widerstand überwinden müssen. Dazu braucht er viel politisches Geschick und viel Überzeugungskraft, aber auch Entschlossenheit. Immerhin kann er dabei die institutionellen Möglichkeiten nutzen, die das Amt inm einräumt, und als parteiunabhängiger Präsident verfügt er über einen breiteren politischen Spielraum als seine Vorgänger. Mit der Wahl des Premierministers, der Zusammensetzung der Regierung, aber auch mit ersten Maßnahmen auf dem Verordnungsweg kann er noch vor der Parlamentswahl im Juni wichtige politische Signale aussenden, für seinen Kurs der Erneuerung werben und seinen politischen Spielraum verbreitern.

Macron weiß: Die ersten Wochen und Monate werden entscheidend sein für seinen politischen Gestaltungsspielraum der kommenden Jahre. Ein erster Lackmustest für seine Entschlossenheit und Durchsetzungsfähigkeit wird die von ihm forcierte, aber höchst konfliktträchtige Reform des Arbeitsrechts sein. Er will schnell handeln, muss aber auch die Konsultationen mit Arbeitgebern und Gewerkschaften ernst nehmen, um seinem eigenen politischen Anspruch gerecht zu werden. Spätestens im Herbst ist eine Kraftprobe mit der Linken und einem Teil der Gewerkschaften, die bereits jetzt gegen die geplante Reform mobilisieren, unausweichlich. Ihr Ausgang kann für den Erfolg der gesamten Präsidentschaft und damit für die Chancen der politischen, wirtschaftlichen und sozialen Erneuerung Frankreichs vorentscheidend sein. 